\title{
A MODEL FOR THE GLOBULAR CLUSTER LUMINOSITY FUNCTION
}

\author{
DEAN E. MCLAUGHLIN AND RALPH E. PUDRITZ \\ Dept. of Physics and Astronomy, McMaster University \\ Hamilton, Ontario L8S $4 M 1$ Canada
}

We develop the idea (Harris \& Pudritz 1994, ApJ, 429, 177) that, like currently forming star clusters and associations, globular clusters (mean mass $\left.\simeq 3 \times 10^{5} M_{\odot}\right)$ were born in the 'cores' of much larger $\left(\sim 10^{8}-10^{9} M_{\odot}\right)$ star-forming complexes which we call 'supergiant molecular clouds,' or SGMCs. The number $N(m)$ of protoclusters at mass $m$ is then determined by a steady-state balance between their growth by core-core collisions, and their self-destruction via the side effects of star formation. This mass spectrum is ultimately passed on to the globular cluster system (GCS) itself, by virtue of the very high star-formation efficiency required to produce a bound stellar cluster from a gaseous core.

The major influence on the shape of the GCS mass spectrum is the ratio $\beta$ of fiducial core disruption and collision timescales. Our models are further characterized by a mass-dependent core lifetime: below a critical mass $m_{\star}$, star formation is too passive to disrupt a core; but above this limit, cores will self-destruct in a finite amount of time. We identify $m_{*}$ with the peak magnitude of the globular cluster luminosity function $[\phi \sim m N(m)]$. Its value and the peak mass $m_{1}$ of the luminosity-weighted luminosity function $\left[\psi \sim m^{2} N(m)\right]$ are then used to fit the observed mass spectra (above $m_{*}$ ) of the Milky Way, M31, and M87 GCSs (see McLaughlin \& Pudritz 1996, ApJ, 456, in press; also Harris, these proceedings).

Our main results are: (1) The ratio $\beta$, and hence the shape of the GCS mass spectrum, is expected to be independent of position within a galaxy. (2) $m_{1}$ varies among GCSs, and is roughly that mass above which a core's collisional growth time is longer than its lifetime. (3) More massive cores in a given SGMC must be shorter-lived; specifically, the data imply that core disruption times scale as $m^{-0.6}$ above $m_{*}$. (4) $\beta$ is significantly larger, and the GCS mass spectrum shallower, in M87 than in the Local Group spirals. This is likely an effect more of environment than of Hubble type alone. 